\title{
Coherent wave activity in Mercury's magnetosheath
}

\author{
Torbjörn Sundberg ${ }^{1 *}$, Scott A. Boardsen ${ }^{2,3}$, David Burgess ${ }^{1}$, and James A. Slavin ${ }^{4}$
}

${ }^{1}$ School of Physics and Astronomy, Queen Mary University of London, London, UK.

2 Goddard Planetary Heliophysics Institute, University of Maryland, Baltimore County, Baltimore, Maryland, USA

${ }^{3}$ Heliophysics Science Division, NASA Goddard Space Flight Center, Greenbelt, Maryland, USA.

${ }^{4}$ Department of Atmospheric, Oceanic and Space Sciences, University of Michigan, Ann Arbor, Michigan, USA

Correspondence to: t. sundberg@qmul.ac.uk

\section{Abstract}

This study presents a statistical overview of coherent wave activity in Mercury's magnetosheath. Lefthanded electromagnetic ion-cyclotron waves are commonly found behind the quasi-perpendicular section of the bow shock, where they are present in $\sim 50 \%$ of the spacecraft crossings of the magnetosheath. Their occurrence distribution maximizes within the magnetosheath, approximately halfway between the bow shock and the magnetopause, and the waves are generally strongly Doppler shifted up to frequencies above the local ion-cyclotron frequency. Downstream of the quasi-parallel shock, the magnetosheath often exhibit large-amplitude pulsations with wave periods around $10 \mathrm{~s}$ and peak-to-peak amplitudes of up to $100 \mathrm{nT}$ that dominate the magnetic field structure. These waves are circularly left-hand polarized with wave vectors in the direction of the local shock normal. The data suggest that they have been generated upstream of the shock and transmitted into the downstream region. Their occurrence rates maximize at the near-parallel shock, where they are present approximately $10 \%$ of the time, and where they also show their largest wave powers. Some evidence is also found of waves with a right-handed polarization in the spacecraft frame. These consist of both whistler waves above the local ion-cyclotron frequency and ion-cyclotron waves propagating against the magnetosheath flow with Doppler shifts exceeding the intrinsic wave frequency, which results in a change in their apparent polarization. These waves are in minority compared to the left-handed observations, which This is the author manuscript accepted for publication and has undergone full peer review but has not been through the copyediting, typesetting, pagination and proofreading process, which may lead to differences between this version and the Version of Record. Please cite this article as doi: 10.1002/JGRA.52042 
indicates a preference for ion-cyclotron waves propagating in the direction of the plasma flow.

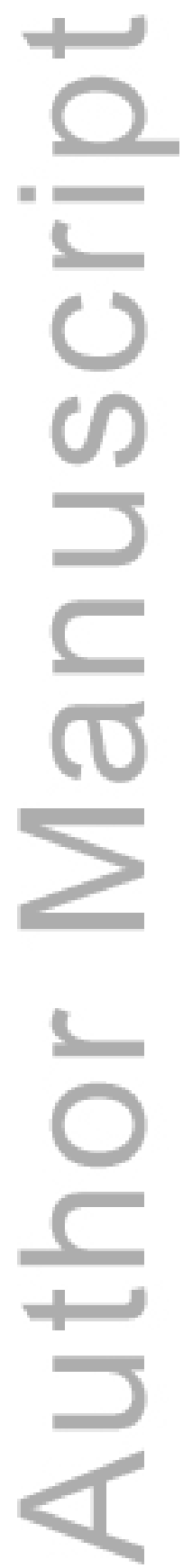

This article is protected by copyright. All rights reserved. 


\section{Introduction}

In-situ observations by the Mariner 10 and MESSENGER (MErcury Surface Space ENvironment GEophyscs and Ranging) spacecraft have revealed that Mercury's magnetosphere is under constant reconfiguration; the system is strongly driven by the large solar wind magnetic fields and densities found in the inner parts of our solar system, and transient and dynamic events are dominant throughout most of the planet's small magnetosphere [e.g., Slavin et al. 2008, 2009, 2010; Ho et al., 2012; Sundberg et al., 2012a, 2012b]. In addition, the planet is host to a wealth of wave activity, ranging from bow shock induced waves upstream and downstream of the shock, electromagnetic ion-cyclotron waves (EMIC) in the magnetosheath, Kelvin-Helmholtz waves at the magnetopause and ion Bernstein waves in the magnetotail [Fairfield and Behannon, 1976; Boardsen et al., 2009; 2010, 2012; Le et al., 2013; Sundberg et al., 2012a, 2013]. Most of these have characteristics different from those observed at Earth, due to factors such as the different solar wind conditions, the comparatively warm plasma in the magnetosphere, and the much smaller scale-size of the system. This study aims to further classify the wave activity present in the magnetosheath, primarily the EMIC mode which is commonly observed in the magnetosheaths of both Mercury and at Earth.

The magnetosheath is an important part of the solar wind-magnetosphere interaction for any planet, since it mediates the parameters and dynamics of the solar wind before it interacts with the magnetopause. In particular, wave activity and turbulence in the magnetosheath can play an important role in the coupling between the solar wind flow and the magnetopause. Wave activity can originate from waves convected through the bow shock from upstream or from waves created at the bow shock and then convected downstream, and unstable particle distribution functions generated at the bow shock can lead to waves driven by instabilities downstream. The flow pattern within the magnetosheath also leads to modifications of the particle distribution functions by compression, field line stretching and magnetic shear [Zwan and Wolf, 1976], and this too can produce waves driven by kinetic instabilities. The wave activity and the role of instabilities in the terrestrial magnetosheath, the best studied example, is reviewed by Schwartz et al. [1996]. Dissipation at the supercritical quasi-perpendicular shock depends on the reflection of a fraction of the incident ions, which produces a gyrating population with a perpendicular temperature anisotropy, and associated wave activity [e.g., McKean et al. 1995]. At low frequencies the relevant instabilities are the ion temperature anisotropy cyclotron instability (producing waves on the electromagnetic ion cyclotron branch) and the mirror instability [e.g., Gary, 1995]. In both cases, the waves driven by the instabilities act to reduce the temperature anisotropy via pitch-angle 
scattering either by cyclotron effects or scattering in the mirror-mode structures. Mirror waves are nonpropagating and compressive in nature, compared to, for parallel propagation, the transverse noncompressional EMIC waves. In the terrestrial magnetosheath there is evidence for both types of wave activity [e.g., Hubert et al., 1998] depending on location relative to the bow shock and magnetopause. The effect of flow compression on instabilities and driven waves has been studied using the hybrid expanding box model by Hellinger and Travnicek [2005]. The magnetosheath downstream of the quasiparallel shock is usually considerably more complex, since transmission of upstream waves convected by the flow gives a major component of the downstream wave activity, especially at intermediate Mach numbers [Krauss-Varban and Omidi, 1991].

The presence of ion-cyclotron waves in Mercury's magnetosheath was determined already by the Mariner 10 mission [Fairfield and Behannon, 1976], and the wave mode has subsequently received a great deal of attention at Earth [e.g., Anderson et al., 1991; Gary, 1992; Anderson and Fuselier, 1993, 1994; Denton et al., 1993]. EMIC waves are characterized by near transverse oscillations with a left-hand polarization, close to circular polarization, and low wave-normal angles. The wave mode is supported below the local proton gyro frequency, $f_{\mathrm{H}+}$, with a subsequent mode transition at the gyro frequency of any of the heavier species that may be present in the plasma, such as $\mathrm{He}^{++}$or $\mathrm{Na}^{+}$[e.g., Gary et al., 1993]. There is also a close relation between the wave frequency, the wave normal angle and the ellipticity, with the waves closer to a cyclotron resonance frequency being more transverse and more circular in their polarization.

Mirror-mode activity has been predicted at Mercury [Herčík et al., 2013], but not yet observed. The mirror mode is a non-propagating wave in the plasma rest frame that are characterized by simultaneous fluctuations in the plasma density and the background magnetic field, where the magnetic field fluctuations are close to linearly polarized in the field-aligned direction. The relative growth of the EMIC and mirror-mode instabilities has been determined as dependent on the plasma $\beta$ and the temperature anisotropy ratio [e.g., Gary 1976, Gary 1992, Yoon 2012], where $\beta$ is the ratio between the thermal and magnetic pressures, with EMIC more prominent in low $\beta$ plasmas. The mirror-mode is the more common process at Earth [e.g., Schwartz et al., 1996], but both wave modes are observed. EMIC waves are typically seen behind low Mach number quasi-perpendicular shocks [Soucek et al., 2015], whereas mirrormode waves dominate for Mach numbers above 7 . The Mirror-mode instability is especially pronounced in the magnetosheath at Jupiter and Saturn [e.g., Erdös and Balogh, 1996] as the solar wind $\beta$ increases 
with the distance from the Sun. In addition, a thick plasma depletion layer is commonly observed outside the dayside magnetopause, which can lead to an even lower $\beta$ environment, on the order of a factor $1 / 5$ or less compared with that that at the bow shock [Gershman et al., 2013]. Some of the best examples of such a low $\beta$ environment can be seen during the interaction with coronal mass ejections [Slavin et al., 2014]. For these reasons, we can expect the low solar wind beta and the weaker bow shock at Mercury to lead to a magnetosheath environment where the ion temperature anisotropy cyclotron instability dominates over the mirror instability.

We here build on the Mariner 10 results by using approximately three Mercury years of MESSENGER data (1 Mercury year is equivalent to 88 Earth days) in order to provide a more thorough view of the different wave modes in the magnetosheath, their characteristics and occurrence frequency. Mode detection of waves in spacecraft data is an intrinsically difficult task [e.g., Schwartz et al., 1996], as the wave observations usually consist of a mixture of wave modes and frequencies, possibly exhibiting non-linear characteristics or effects due to plasma inhomogeneities. Wave propagation characteristics such as the wave vector and the Doppler shift are also difficult to determine from single spacecraft observations. In addition, reliable determination of low-frequency waves requires long periods with quiet magnetic field conditions, something which can often be difficult to find at Mercury due to the strong ion-kinetic turbulence present in the magnetosheath [Uritsky et al., 2011]. For that reason, we will here as a first step focus on the classification of long-lived, coherent waves that are easily detectable and unambiguous in their interpretation. This analysis will therefore primarily focus on EMIC waves, whereas a full investigation of mirror-mode waves may require a separate effort.

\section{Wave Modes}

We have performed a statistical survey of coherent waves during all magnetosheath crossings recorded by the MESSENGER Magnetometer Instrument in 2011. After the orbit insertion on March 22, MESSENGER followed an eccentric orbit around the planet with periapsis and apoapsis of $\sim 1.1$ and $7 R_{\mathrm{M}}$ (where $R_{\mathrm{M}}$ denotes Mercury's radius, $1 R_{\mathrm{M}}=2440 \mathrm{~km}$ ), respectively. The orbit precesses around the equatorial plane over the Mercury year; Figure 1 shows the spacecraft orbit in the noon-midnight and dawn-dusk planes, together the approximate location of the magnetosheath crossings associated with the respective orbital position. As Mercury's distance from the Sun varies in between 0.31-0.47 AU over the Mercury year, there is a co-dependence between the orientation of the spacecraft's orbit and the planet's orbital phase; MESSENGER's apoapsis is on the dusk side at perihelion and on the dawn side at 
aphelion. As the solar wind parameters change with radial distance from the Sun, this orbital dependence therefore influences the average observed properties of the solar wind environment, including the particle density and temperature, and the magnetic field direction and magnitude.

For our magnetosheath intervals, we have limited the analysis to the outermost magnetopause and the innermost bow shock crossings, using the data set provided by Winslow et al. [2013]. This gives the most conservative estimate of the magnetosheath, and thereby avoids introducing any spurious wave detections in either the magnetosphere or the solar wind.

Using a short-windowed Fourier transform of the magnetosheath data, we calculate the coherence between the maximum and intermediate variance components of the magnetic field as a function of frequency and time, using time intervals of $30 \mathrm{~s}$. The Messenger magnetometer generally samples at 20 $\mathrm{Hz}$ [Anderson et al., 2007], which limits the frequency span to $0.05-10 \mathrm{~Hz}$. This should be sufficient for our purposes as the typical ion-cyclotron periods in the magnetosheath is expected to be on the order of 0.5-2 Hz [Fairfield and Behannon, 1976]. The coherence analysis gives an estimate of how stable the two components are in phase in a particular frequency span. The analysis results in a detection of circular and elliptically polarized waves, and we expect it to provide a reliable classification of proton-driven EMIC waves. Sodium ion-cyclotron waves may potentially also be present in the magnetosheath at lower frequencies, but they may be more difficult to detect as they require long periods of observation when the level of background fluctuations is low in comparison to the wave amplitude, criteria which are rarely met. Linearly polarized waves such as mirror-mode waves will not be detected by the analysis method, these require a separate effort, and the full analysis of linear waves is not included in the scope of this paper.

Following the coherence analysis, we apply a 2-dimensional filter on the calculated coherence matrix, smoothing the data over both frequency and time, and we use a blob-coloring routine to mark regions where the coherence exceeds the threshold value of 0.5. In order to classify as a proper wave event, we also require that the coherence exceeds the threshold value for least 4 analysis periods, i.e., showing a minimum of 120 seconds of coherent wave activity. For each such instance we determine the average frequency, perpendicular and parallel power-spectral densities $\left(P_{\perp}\right.$ and $\left.P_{\|}\right)$, and ellipticity compared to the background magnetic field over the wave interval. These values are then used for the wave mode classification and the statistical analysis. It should be noted here that the values are determined in the spacecraft frame, and a proper determination of the Doppler shift is required in order to determine the 
true wave frequency and polarization. Figure 2 shows the ratio between the compressive and transverse power components against the total power-spectral density (PSD) in the $0.05-10 \mathrm{~Hz}$ frequency band for all detected wave events, with each wave event color-coded by their ellipticity with left-hand polarized waves marked in blue and right-hand waves in red. It is apparent here that a few different wave modes are present in the data: A majority of the observations are left-hand polarized highly transverse waves with a PSD in the region of $1-20 \mathrm{nT}^{2} / \mathrm{Hz}$. This group is predominantly represented by proton EMIC waves, and will be studied in more detail in Section 2.1. A second wave group is found in the upper domain of the PSD axis, with power densities exceeding $\sim 300 \mathrm{nT}^{2} / \mathrm{Hz}$. These are left-hand polarized and partly compressional. We believe that these waves are associated with the quasi-parallel bow shock, and they will be investigated in Section 2.2. There is also a third sporadic population of waves in the lower righthand region, i.e., partly compressional waves with low PSDs. A large portion of these are right-hand polarized, and they will be studied in Section 2.3.

\subsection{Electromagnetic ion-cyclotron waves}

Observations reported from Mariner 10's first flyby of Mercury showed a strong wave activity in the duskside magnetosheath, primarily in the $\sim 1-3 \mathrm{~Hz}$ frequency band [Fairfield and Behannon, 1976]. The reported waves had a clear left-hand polarization, were strongly transverse, and $\sim 1-2 \mathrm{~Hz}$ above the local proton gyro frequency, $f_{\mathrm{H}+}$. As the left-hand polarized wave mode only exists at frequencies below $f_{\mathrm{H}+}$, the results were interpreted as EMIC waves Doppler shifted by the magnetosheath flow to a higher frequency span. Using rough estimates of the wave vector and the plasma flow velocity, Fairfield and Behannon [1976] derived approximate Doppler shifts and showed that the observations and theoretical estimates were reasonably well matched. We here define EMIC waves as highly transverse left-hand polarized waves with $P_{\perp}>5 P_{\|}$. In addition, we also impose a restriction on the total wave power, which should be less than $\sim 65 \mathrm{nT}^{2} / \mathrm{Hz}$. This limit is the mean wave power averaged over all wave encounters, and it seems to bea reasonable upper limit for the EMIC. Both of these constraints are shown by the dashed lines in Figure 2. This classification provides a homogenous data set with a median PSD of $\sim 5 \mathrm{nT}^{2} / \mathrm{Hz}$, which allows us to investigate the EMIC characteristics statistically.

One example of a magnetosheath passages that contained strong EMIC wave activity, recorded on 19 June 2011, will here be described in detail. The passage took place after an outbound crossing of the southern tail lobe, in between 12:40 and 13:15 UTC. An overview of the spectral properties of the magnetosheath crossing is given in Figure 3, which shows the evolution of $P_{\|}, P_{\perp}$, the ellipticity, $\varepsilon$, and the 
coherence, $c$, with the magnetopause on the left to the bow shock on the right. For the ellipticity, $\varepsilon=1$ corresponds to circular right-hand polarization, $\varepsilon=-1$ to left-hand polarization, and $\varepsilon=0$ to linear polarization. The spacecraft position is given in Mercury solar magnetic coordinates, which are centered at the planetary dipole, $0.2 R_{\mathrm{M}}$ north of the planetary center [e.g., Anderson et al., 2011]. Coherent, lefthand polarized waves with a dominant perpendicular power component were present during a period of 12 minutes, $12: 54-13: 06$ UTC. The wave frequency at the start of the interval was close to the local $f_{\mathrm{H}+}$ at $\sim 1 \mathrm{~Hz}$, but it gradually increased over the time span of the observation up to a final frequency of $\sim 1.4$ $\mathrm{Hz}$, whereas $f_{\mathrm{H}+}$ remained stable at $1 \mathrm{~Hz}$ throughout the magnetosheath. The waves were close to circularly polarized $(\varepsilon<-0.8)$ and had a wave normal angle of around $10-15^{\circ}$, determined from a comparison between the field-aligned and minimum variance directions over short intervals. Figure 4 shows an excerpt of the wave pattern in between $12: 58: 40$ and 12:58:48 which highlights some the main characteristics. There are clear sinusoidal oscillations in the two transverse components with an amplitude of $\sim 10 \mathrm{nT}$, but the amplitude varies in between each wave encounter, which is typical for waves at marginal stability as they propagate through regions of both growth and decay [e.g., Cairns and Grubits, 2001]. Their polarization remained circular throughout the interval, as is seen in the associated hodograms, shown in the right-hand panels of Figure 4.

Both the wave number, $\bar{k}$, and magnetosheath velocity are unknown, but an estimated maximum limit of $k$ at $\sim 0.02 \mathrm{~km}^{-1}$ was given by Fairfield and Behannon [1976], which consequently gives a Doppler shift of $<\sim 1 \mathrm{~Hz}$, assuming a magnetosheath velocity of $320 \mathrm{~km} / \mathrm{s}$. The direction of $\bar{k}$ is also undetermined, but as the wave frequency increases toward the bow shock, in line with a gradual increase of the magnetosheath flow velocity as the spacecraft gets further away from the magnetopause, it is reasonable that the increase in flow velocity leads to and increased Doppler shift. In addition, the magnetic field draping within the magnetosheath may also contribute to a more parallel alignment of the wave vector (which is assumed to be primarily field aligned) and the plasma bulk flow. This observed increase in wave frequency toward the bow shock is also a feature typical of most EMIC wave events found in this study. If we assume that the wave frequency in the plasma rest frame is stable at a fraction $\alpha$ of the ion cyclotron frequency and that all parameters apart from the magnetosheath flow velocity remain stationary during the observation, we can derive estimates of the Doppler shift through the relation $\omega_{\mathrm{sc}}=\bar{k} \cdot \bar{v}+\alpha \Omega_{\mathrm{H}+\text {, }}$ where $\omega_{s c}$ is the angular frequency in the spacecraft frame, $\bar{v}$ is the plasma flow velocity, $\alpha$ is a scaling factor, and $\Omega_{\mathrm{H}_{+}}$is the cyclotron angular frequency of the protons. If we further assume a lower limit of $\alpha=0.6$, this gives a Doppler shift ranging between $0.4-0.8 \mathrm{~Hz}$ for the EMIC period, i.e., corresponding to an 
increase in the magnetosheath flow velocity of an factor of 2 between the inner and outer edge of the EMIC interval. In this case, an assumed flow velocity increase from 200 to $400 \mathrm{~km} / \mathrm{s}$ corresponds to $k=$ $0.013 \mathrm{~km}^{-1}$. These values are on the border of what might be considered physically plausible, and they indicate that the true nature of the wave propagation may be more intricate than what is captured by the simple model presented here.

\subsection{Large-amplitude waves}

The spectral overview in Figure 2 shows that a fraction of the wave detections are large-amplitude lefthand polarized waves with varying ratio between the compressional and transverse power-spectral components. These waves have a much lower frequency than the EMIC waves discussed previously, on the order of $0.1 \mathrm{~Hz}$, and all of them are found below the local ion-cyclotron frequency. It is difficult to set an exact definition for this group in terms of PSD, ellipticity and frequency, but we argue that a majority of the events in the upper regions of Figure 2 (i.e., PSDs exceeding $300 \mathrm{nT}^{2} / \mathrm{Hz}$ ) are similar in nature, and that they provide sufficient statistics in order to draw some conclusions on their characteristics. One such example from 22 July 2011 is shown in Figure 5, where large-amplitude waves are present from the bow shock to half way into the magnetosheath. In this case, the bow shock itself is not actually associated with a change in the DC component of the magnetic field, but the increase in $|\mathrm{B}|$ from 20 to $60 \mathrm{nT}$ is due to a change in amplitude of the wave envelope alone. The wave normal angle is stable throughout the wave interval at 40-45 degrees, as is the wave amplitude. Figure 6 shows the magnetic field components during a 2-min subset of the wave interval. Although the wave amplitude stays roughly constant throughout this period, there is a certain variation in the wave period over the interval, with the shortest cycles being about $7 \mathrm{~s}$, and the longest roughly $12 \mathrm{~s}$. The associated hodograms, displayed in the panels on the right, show that the wave is approximately circular in the maximum and intermediate variance components. As the total magnetic field also remains relatively steady throughout the wave observation, these waves are not reconcilable with the mirror mode.

Overall, the wave properties are reminiscent of the magnetosheath pulsations reported by Sundberg et al. [2013]. In that study, it was shown that the reformation of a quasi-parallel shock can drive strong magnetosheath and magnetospheric wave activity at Mercury. Similar observations have since then also been made at Venus, where Shan et al. [2014] observed a transmission of ULF waves from the upstream to the downstream region. Approximately half of the wave events also show pulsations in a similar frequency span but at much lower amplitudes in the region immediately upstream of the shock, which is 
also indicative of an upstream source for this wave mode, and we can interpret these results following the simulation results by Krauss-Varban and Omidi [1991], who showed that the transmission of waves from upstream to downstream can become possible at intermediate Mach number shocks.

\subsection{Right-hand polarized waves:}

The right-hand polarized waves seen in Figure 2 cover a broad range of $P_{\|} / P_{\perp}$, and they generally have lower PSDs than the EMIC. Figure 7 shows the frequency distribution of the right-hand waves, normalized to the local proton cyclotron period. The distribution has two clearly defined peaks, one below the cyclotron period, and one at higher frequencies. We interpret these as two different wave families, with the lower period waves being related to Doppler-shifted EMIC waves, and the higher period waves being Whistler-mode waves which are not limited by the proton cyclotron frequency. We note that both of these wave types are much less frequent than the left-hand EMIC waves, and, with their lower average wave powers, they are also generally less pronounced in the data.

An example of a low-frequency right-hand polarized wave observed on 15 October 2011 is shown in Figure 9. The figure follows the same format as Figure 3, with the outermost magnetopause crossing on the left and the innermost bow shock on the right. These recordings are similar to those presented for the June 19 event. A period of intense EMIC wave activity was observed from 15:40 to 15:50 UTC. The wave frequency at the start of this interval was close to the local $f_{\mathrm{H}_{+}}$at $\sim 1.8 \mathrm{~Hz}$, but it experienced a gradual increase over the time span of the observation up to a final frequency of $\sim 2.1 \mathrm{~Hz}$, whereas $f_{\mathrm{H}+}$ remained stable throughout this interval. Simultaneous with the left-handed waves, a population of righthand polarized waves appeared at lower frequencies, sporadically present from 15:45 and all the way to the bow shock crossing at 16:15 UTC. These waves were less coherent than the left-hand polarized population, they had a lower average $P_{\|} / P_{\perp}$, but they showed a similar frequency shift toward higher frequencies over the observation interval, and the wave power was on the order of a third of that of the left-handed waves. The wave pattern was strongest and most coherent after the left-hand population had diminished. Both wave populations were close to circularly polarized, with wave normal angles around $15^{\circ}$. For the left-handed waves, the quasi-circular polarization suggests that the wave frequency in the plasma reference frame should be at or slightly below $f_{\mathrm{H}^{+}}$, and that the waves are subsequently Doppler-shifted to the higher frequency span. As noted earlier, the wave propagation is intrinsically hard to determine, but the low wave-normal angles recorded indicate that $\bar{k}$ and $\bar{v}$ are nearly parallel, as the 
magnetic field direction is close to that expected for the plasma flow in the region. This is most likely the sign of two counter-propagating wave populations near the $\Omega_{\mathrm{H}_{+}}$frequency, Doppler shifted into a highfrequency left-hand mode and a low-frequency right-hand mode. In order for the second population to be observed as right handed, this requires that the Doppler shift exceeds the intrinsic wave frequency.

With the assumption that the wave properties and the Doppler shifts are similar for both populations, apart from the wave vectors being oppositely directed, we can estimate what the wave frequency and Doppler shift may have been. We have selected a 2-minute wave interval centered at 15:49 UTC for analysis purposes. Similar to the earlier analysis, we assume that the wave frequency in the plasma rest frame is stable at a fraction $\alpha$ of the ion cyclotron frequency, and that the Doppler shift from is equal but opposite for the two wave populations. As the main frequency peaks in this interval were located at $\sim 2.1$ and $\sim 0.6 \mathrm{~Hz}$ for the left-hand and right-hand population, respectively, and the ion cyclotron frequency was $1.9 \mathrm{~Hz}$, this gives us an approximate Doppler shift of $\sim 1.35 \mathrm{~Hz}$ and $\alpha \approx 0.4$. If we further assume a magnetosheath velocity of $400 \mathrm{~km} / \mathrm{s}$ and a perfectly flow-aligned wave vector, this gives a wave number of $k \approx 0.02 \mathrm{~km}^{-1}$. Similar to the event analyzed above, this analysis indicates a lower wave frequency and higher Doppler shift than would be expected from the ellipticity and the wave normal angle observed.

\section{Statistics}

We have performed a thorough investigation of the wave occurrence patterns in order to better understand the processes driving the wave generation. For each magnetosheath encounter we estimate the current IMF conditions with the magnetic field measurements taken just upstream of the bow shock, averaged over a 15 minute time period. We also estimate the position within the magnetosheath for each wave encounter relative to the magnetopause and the bow shock and the angle between the IMF and the local shock normal at the MESSENGER bow shock crossing, $\theta_{B N}$. Quasi-parallel shocks are defined by $\theta_{B N}<45^{\circ}$, and quasi-perpendicular as $\theta_{B N}>45^{\circ}$. These results are summarized in Figure 9. It is here evident that the EMIC waves and the large-amplitude waves have completely different characteristics in terms of their occurrence distribution and IMF dependence. The EMIC waves are almost exclusively found behind a locally quasi-perpendicular shock; less than $15 \%$ are found for $\theta_{B N}<45^{\circ}$, and there is a strong bias toward nearly perpendicular geometries. Almost 50\% of the crossings of perpendicular shocks shows some EMIC activity. This agrees with our expectations as these are the regions where the perpendicular temperature anisotropy is expected to be at its largest. The EMIC occurrence distribution peaks roughly halfway between the magnetopause and the bow shock, with a slight shift toward the shock. The 
occurrence distribution also goes down to approximately zero at both the inner and outer boundary of the magnetosheath. Typical wave amplitude to background magnetic field ratios ( $\delta \mathrm{B} / \mathrm{B})$ are 0.05-0.15. The results for the large-amplitude waves, which have a $\delta B / B$ of $0.4-1$, are in stark contrast to what is found for the EMIC waves. These are instead clearly biased toward the quasi-parallel side of the magnetosheath, with a majority of the events observed for $\theta_{\mathrm{BN}}<45^{\circ}$. They are also predominantly found in the immediate downstream region of the shock. These detections represent at around $5 \%$ of all quasiparallel crossings, and $10 \%$ of those with $\theta_{B N}<25^{\circ}$. This clustering of large-amplitude waves at the quasiparallel shock is even more prominent when using the $\theta_{B N}$ at the subsolar point rather than the local value, which possibly indicates that these large-amplitude pulsations are a global phenomenon at the quasi-parallel shock.

The relation between the wave propagation direction and the wave power is shown in Figure 10. Here, the left-hand plot shows the occurrence rate as a function of the angle between the wave normal (estimated from the minimum variance direction) and the local bow shock normal, $\theta_{\mathrm{kN}}$. These are heavily biased toward parallel and anti-parallel orientations, with an occurrence maximum for weakly oblique shocks. This means that the magnetic field perturbations of the large-amplitude waves are generally in the plane of the local shock, and that the waves most likely propagate away from the bow shock approximately in the direction of the shock normal. The right-hand panel in Figure 10 shows how this agreement between the wave propagation direction and the shock normal influences the wave power: the closer the alignment, the stronger the waves. This again signifies that large-amplitude waves are a global phenomenon in the magnetosheath, with an epicenter at the exactly parallel shock and the wave power decreasing the further away from quasi-parallel conditions. The natural measure for this comparison would be to look at the wave power in comparison to the local $\theta_{\mathrm{BN}}$. This shows a similar trend with decreasing PSDs with increasing angles, however the connection is somewhat less clear, probably due to the additional errors that are introduced in the calculation of the local bow shock normal. This is the reason why we rather opt for the format shown in Figure 10. Although not shown in the plot, an indirect dependence of the PSD on $\theta_{\mathrm{BN}}$ is also evident from the $\theta_{\mathrm{kN}}$ and $\theta_{\mathrm{kB}}$ distributions.

\section{Discussion}

That the generation of EMIC-waves takes place almost exclusively behind the quasi-perpendicular bow shock is expected due to the anisotropic ion population in this region, however, the spatial distribution 
within the magnetosheath shown in Figure 9 requires a little more thought. Although the temperature anisotropy is presumably generated at the shock itself, this does not necessarily imply that the occurrence frequency would peak in the immediate vicinity of the shock. Further compression and evolution of magnetosheath downstream can lead to increased temperature anisotropies downsteam, and if the growth time of the instability is sufficiently slow and the life span of the waves is relatively long, this will also shift the wave occurrence distribution away from the bow shock. In addition, the instability may initially generate a range of wave instances, of which one eventually becomes the dominant mode. All of these factors may explain why the distribution peaks within the magnetosheath rather than at the bow shock. We can also conclude that the source region of the EMIC waves is locally distributed, with the waves going through cycles of growth and decay as they propagate downstream. The spatial distribution shown may also be affected by orbital effects, as the bow shock crossings regularly take place at lower latitudes than the magnetopause crossings. We believe that this influence should be minor, and that it should not affect the overall distribution pattern.

There is a clear trend towards positively Doppler shifted waves in the data, i.e., waves propagating in the same direction as the plasma flow, as described in Section 2.3. We would normally not only expect to see EMIC waves that are positively Doppler shifted to frequencies above $f_{\mathrm{H}+}$, but also waves that are propagating against the magnetosheath flow in the plasma frame, thus experiencing a negative Doppler shift in the spacecraft frame. This would either result in low-frequency EMIC observed as left-handed, or if the Doppler shift exceeds the intrinsic wave frequency, EMIC waves that are observed as right-handed. Given the large influence of the Doppler shift and the sparse occurrence of low frequency left-hand polarized EMIC, we would thus expect right-hand polarized waves to be common at frequencies below the local $f_{\mathrm{H}+}$ However, in comparison to the left-hand polarized waves, the right-hand detections are relatively rare and both less coherent and less transverse than the left-hand polarized observations. This may either be a consequence of the negatively Doppler shifted waves being more difficult to observe, as lower frequency waves require longer periods of undisturbed conditions which makes them more prone to low-frequency noise and changes in the magnetosheath magnetic field, or it could be an effect of the driving instability, since at the quasi-perpendicular shock the reflected-gyrating downstream ions gain a velocity component parallel to the magnetic field, so that the distribution function is not perfectly symmetrical in the field-parallel direction [Liu et al., 2005]. The solar wind ion distribution often includes secondary proton and alpha particle streams that propagate faster than the bulk plasma, which may also contribute to anisotropic ion distributions downstream [e.g., Marsch et al., 1982a, 1982b]. For these 
reasons, the growth rates for waves with $\mathrm{k}$ parallel and anti-parallel to B could thus be slightly different. A third explanation is that waves propagating against the plasma flow either experience an increased dampening due to a longer traveling time from the source region, or that they are lost upon interaction with the bow shock. Waves that are generated in the immediate downstream region may well propagate back toward the shock where they will dissipate, reflect, and/or mode convert.

Although the analytical treatment of the two EMIC events in Section 2 is simplistic, it still gives an indication that the increase in wave frequency with radial distance often is larger than what is expected. There is evidence for overall large Doppler shifts and low intrinsic wave frequencies compared to the local ion cyclotron frequency, something which is not expected from the relatively circular appearance of the wave polarization and the low compressional components of the waves. Hopefully, a more detailed analysis of the wave properties should be able to resolve this issue.

Regarding the bow shock induced pulsations, they seem to be a typical feature at the intermediate Mach number parallel shock. If the Mach number is sufficiently weak and the fraction of backstreaming ions may not be sufficient to enable self-reformation of the quasi-parallel shock, and a more steady-state transition region may develop than what is normally the case at the quasi-parallel shock. Upstream generated ULF wave field can be injected into the magnetosheath without much dissipation at the shock, possibly with an associated mode conversion from fast-mode waves upstream to Alfven-mode waves downstream of the shock due to the different drift speeds within the upstream and downstream regions, as shown by Krauss-Varban and Omidi [1991]. In the events analyzed here, the downstream waves observed often show peak-to-peak amplitudes of up to $100 \mathrm{nT}$, a factor of $\sim 3$ or more of the upstream wave field. This increase may in part be explained by a decrease of the propagation velocity across the shock together with the conservation of magnetic wave energy, however, as not all events show clear wave activity in the immediate upstream region, this is likely not the only explanation. A global model of the shock interface may be required in order to fully understand the wave generation and the wave propagation patterns. In many of the events observed, the large-amplitude waves extend almost half-way into the magnetosheath without much reduction in the wave amplitude, and the inner edge of the wave train is often associated with an abrupt edge rather than a slow steady decrease in amplitude. This can be seen for example in Figure 4, and it may be an indication that the spacecraft entered into a region of the magnetosheath which is primarily controlled by the quasi-perpendicular magnetosheath, or that the solar wind conditions changed. Given the low apparent dissipation rates of these waves and the relatively small 
scale size of the Mercury's dayside it is possible that the entire subsolar magnetosheath could develop a resonant interaction between the bow shock and the magnetopause when the IMF is near radial, with waves incident from the bow shock being transmitted and/or reflected at the magnetopause, similar to what was discovered by Sundberg et al. [2013].

\subsection{Pick-up ion EMIC}

Strong pickup-ion-driven wave activity has frequently been observed at both Mars and Venus [e.g., Barabash et al., 1991; Russell et al., 2006, Delva et al., 2008] as well as around moons and comets [e.g., Glassmeier et al., 1989; Warnecke et al., 1997]. Given the large presence of sodium and sodium ions in Mercury's magnetosphere [McClintock et al., 2009; Zurbuchen et al., 2011] we would similarly expect to see pickup-ion activity also in Mercury's magnetosheath, but in this study we have found no observations that can be reliably attributed to heavy ion pick-up waves. The proper detection of $\mathrm{Na}^{+}$waves at Mercury may be impeded both by their low frequencies and the unknown contribution of the Doppler shift. As the ion cyclotron frequency scales with the particle mass, EMIC waves driven by $\mathrm{Na}^{+}$pick-up ions would appear at a fraction $1 / 23$ of the local $f_{\mathrm{H}_{+}}$in the plasma rest frame, this requires a long period of undisturbed measurements in order to detect and properly characterize such waves and low-frequency disturbances are likely to interfere with the wave detection. A similar conclusion was reached by Boardsen and Slavin [2007], based on a study of Mariner 10 magnetometer data. In addition, if the waves are significantly Doppler shifted, it is difficult to unambiguously connect an observed wave event to a specific ioncyclotron frequency. As $\mathrm{H}^{+}$waves are abundant in the magnetosheath, these may impede the proper detection of strongly Doppler-shifted heavy ion EMIC.

\subsection{Mirror-mode structures}

For an unambiguous identification of mirror structures, the magnetic field data should show an anticorrelation with the ion density measurement. Given that the wave period likely is shorter than the sampling period of the Fast Imaging Plasma Spectrometer Instrument on MESSENGER, such a classification may be even more difficult to perform. For these reason, we conclude that the investigation of their occurrence patterns deserves a full treatment separate from the analysis presented here. Automated detection algorithms of mirror-mode waves have been successfully implemented at Jupiter and Saturn, where the overall average background level in the magnetic field is compared to that of local dips and peaks. Although these may be efficient algorithms at the outer planets where the magnetosheath is relatively quiet and mirror-mode structuring is the dominant features in the data, they 
are not necessarily suitable for mirror-mode detection at Mercury where the turbulent background conditions introduce both high- and low-frequency noise that complicate successful detection. From a first overview, mirror-mode structures seem to be relatively rare in the Mercury's magnetosheath, and they are not dominating the magnetic field structure as has been observed at Jupiter and Saturn.

We believe that none of the events reported here can be classified as a mirror-mode encounter, as the mirror-mode is compressional instability and the events detected here are primarily transverse, with a maximum $P_{\| l} / P_{\perp} \approx 2$. This is partly a consequence of the wave detection algorithm, as the requirement of a large degree of coherence between the maximum and intermediate variance components effectively excludes linearly polarized wave modes from the data set. In addition, the mirror mode is not a wave mode in the same sense as the EMIC, but that they are rather non-propagating structures that advect with the background plasma flow, with local depressions and/or peaks in the magnetic field, anticorrelated with the background ion density. For this reason, there may not be an inherent wave frequency associated with the observations, as it depends on the scale size of the structures and the background flow velocity, and mirror mode waves can develop both quasi-sinusoidal and nonperiodic structures [e.g., Bavassano Cattaneo et al., 1998], and in the case of large departure from a uniform periodicity, this further invalidates the coherence analysis.

\section{Summary and conclusions}

In this study, we report the detection of EMIC waves, large-amplitude waves at quasi-parallel bow shock, and whistler waves within Mercury's magnetosheath. The EMIC are by far the most abundant wave population in the magnetosheath. These are primarily observed as strongly Doppler shifted left-hand polarized waves near or above the ion-cyclotron frequency. These Doppler shifts are often larger than expected from simple models. A few events are found with negatively Doppler-shifted waves with a righthand polarization in the spacecraft frame, these are propagating against magnetosheath flow, but they are few in comparison to the left-hand waves, which indicates that there is a preference for EMIC waves propagating in the direction of the flow. This may be due to the interaction with the bow shock, that they experience a larger damping over time, or a preference in the generation mechanism. The EMIC waves are almost exclusively confined to the region behind the quasi-perpendicular bow shock.

The large-amplitude waves can have amplitudes of up to 100 nT peak-to-peak, and they are observed almost exclusively downstream of the quasi-parallel bow shock. The wave normals are generally aligned 
with the bow shock normal, something which explains the observed spread in the magnetic compression ratio, which then depends on the angle between the downstream magnetic field and the bow shock normal. The waves are most commonly found in the outer parts of the magnetosheath, near the bow shock itself, but they can be present up to the midway point between the bow shock and the magnetopause. They also experience a decrease in PSD away from the exactly parallel shock, which seems to be their main point of origin. The results presented here show that these types of pulsations may be very common at Mercury, present approximately $10 \%$ of the time at the parallel bow shock. They can significantly affect the magnetosheath region downstream of the quasi-parallel shock, and potentially also generate low-frequency pulsations within the magnetosphere.

\section{Acknowledgements}

We thank MESSENGER mission, the MESSENGER Magnetometer Instrument team, and the Planetary Data System (https://pds.nasa.gov) for providing the data used in this study. Parts of this work has been funded by the European Commission's Seventh Framework Programme FP7 under the grant agreement SHOCK (project number 284515).

\section{References}

Anderson, B. J., S. A. Fuselier, and D. Murr (1991), Electromagnetic ion cyclotron waves observed in the plasma depletion layer, Geophys. Res. Lett., 18(11), 1955-1958, doi:10.1029/91GL02238.

Anderson, B. J., and S. A. Fuselier (1993), Magnetic Pulsations from 0.1 to $4.0 \mathrm{~Hz}$ and Associated Plasma Properties in the Earth's Subsolar Magnetosheath and Plasma Depletion Layer, J. Geophys. Res., 98(A2), 1461-1479, doi:10.1029/92JA02197.

Anderson, B. J., and S. A. Fuselier (1994), Response of thermal ions to electromagnetic ion cyclotron waves, J. Geophys. Res., 99(A10), 19413-19425, doi:10.1029/94JA01235.

Anderson, B. J., M. H. Acuña, D. A. Lohr, J. Scheifele, A. Raval, H. Korth, and J. A. Slavin (2007), The Magnetometer instrument on MESSENGER, Space Sci. Rev., 131, 417-450.

Anderson, B. J., C. L. Johnson, H. Korth, M. E. Purucker, R. M. Winslow, J. A. Slavin, S. C. Solomon, R. L. McNutt, Jr., J. M. Raines, and T. H. Zurbuchen (2011), The global magnetic field of Mercury from MESSENGER orbital observations, Science, 333, 1859-1862.

Auer, P. L., R. W. Kilb, and W. F. Crevier (1971), Thermalization in the Earth's bow shock, J. Geophys. Res., 76(13), 2927-2939, doi:10.1029/JA076i013p02927. 
Barabash, S., E. Dubinin, N. Pissarenko, R. Lundin, and C. T. Russell (1991). Picked-up protons near Mars: Phobos observations. Geophys. Res. Let., 18, 1805-1808.

Bavassano Cattaneo, M. B, C. Basile, G. Moreno, and J. D. Richardson (1998), Evolution of mirror structures in the magnetosheath of Saturn from the bow shock to the magnetopause, J. Geophys. Res., 103(A6),11961-11972, doi:10.1029/97JA03683.

Boardsen, S.A., and J.A. Slavin (2007), Search for pick-up ion generated Na+ cyclotron waves at Mercury, Geophys. Res. Lett., 34, L22106, doi:10.1029/2007GL031504

Boardsen, S. A., J. A. Slavin, B. J. Anderson, H. Korth, and S. C. Solomon (2009), Comparison of ultra-lowfrequency waves at Mercury under northward and southward IMF, Geophys. Res. Lett., 36, L18106, doi:10.1029/2009GL039525.

Boardsen, S. A., T. Sundberg, J. A. Slavin, B. J. Anderson, H. Korth, S. C. Solomon, and L. G. Blomberg (2010), Observations of Kelvin-Helmholtz waves along the dusk-side boundary of Mercury's magnetosphere during MESSENGER's third flyby, Geophys. Res. Lett., 37, L12101, doi:10.1029/2010GL043606.

Boardsen, S. A., J. A. Slavin, B. J. Anderson, H. Korth, D. Schriver, and S. C. Solomon (2012), Survey of coherent $\sim 1 \mathrm{~Hz}$ waves in Mercury's inner magnetosphere from MESSENGER observations, J. Geophys. Res., 117, A00M05, doi:10.1029/2012JA017822.

Cairns, I. H, and K. A. Grubits (2001), Stochastic growth of ion cyclotron and mirror waves in Earth's magnetosheath, Phys. Rev. E, 64, 056408, doi: 10.1103/PhysRevE.64.056408

Crooker, N. U., and G. L. Siscoe (1977), A mechanism for pressure anisotropy and mirror instability in the dayside magnetosheath, J. Geophys. Res., 82(1), 185-186, doi:10.1029/JA082i001p00185.

Delva, M., T. L. Zhang, M. Volwerk, W. Magnes, C. T. Russell, and H. Y. Wei (2008), First upstream proton cyclotron wave observations at Venus, Geophys. Res. Lett., 35, L03105, doi:10.1029/2007GL032594.

Denton, R. E.,, M. K. Hudson, S. A. Fuselier, and B. J. Anderson (1993), Electromagnetic ion cyclotron waves in the plasma depletion layer, J. Geophys. Res., 98(A8), 13477-13490, doi:10.1029/93JA00796.

Erdős, G., and A. Balogh (1996), Statistical properties of mirror mode structures observed by Ulysses in the magnetosheath of Jupiter, J. Geophys. Res., 101(A1), 1-12, doi:10.1029/95JA02207.

Fairfield, D. H., and K. W. Behannon (1976), Bow Shock and Magnetosheath Waves at Mercury, J. Geophys. Res., 81(22), 3897-3906, doi:10.1029/JA081i022p03897.

Gary, S. P. (1992), The Mirror and Ion Cyclotron Anisotropy Instabilities, J. Geophys. Res., 97(A6), 85198529, doi:10.1029/92JA00299.

Gary, S. P. (1993), Theory of space plasma microinstabilities, Cambridge university press. Cambridge, UK. 
Gary, S. P., M. D. Montgomery, W. C. Feldman, and D. W. Forslund (1976), Proton temperature anisotropy instabilities in the solar wind, J. Geophys. Res., 81(7), 1241-1246, doi:10.1029/JA081i007p01241.

Gary, S. P., S. A. Fuselier, and B. J. Anderson (1993), Ion anisotropy instabilities in the magnetosheath, J. Geophys. Res., 98(A2), 1481-1488, doi:10.1029/92JA01844.

Gershman, D. J., J. A. Slavin, J. M. Raines, T. H. Zurbuchen, B. J. Anderson, H. Korth, D. N. Baker, and S. C. Solomon (2013), Magnetic flux pileup and plasma depletion in Mercury's subsolar magnetosheath, J. Geophys. Res., 118, doi:10.1002/2013JA019244.

Hellinger, P., and P. Trávníček (2005), Magnetosheath compression: Role of characteristic compression time, alpha particle abundance, and alpha/proton relative velocity, J. Geophys. Res.,110, A04210, doi:10.1029/2004JA010687.

Herčík, D., P. M. Trávníček, J. R. Johnson, E.-H. Kim, and P. Hellinger (2013), Mirror mode structures in the asymmetric Hermean magnetosheath: Hybrid simulations, J. Geophys. Res. Space Physics, 118,405417, doi: 10.1029/2012JA018083

Ho, G. C., S. M. Krimigis, R. E. Gold, D. N. Baker, B. J. Anderson, H. Korth, J. A. Slavin, R. L. McNutt Jr., R. M. Winslow, and S. C. Solomon (2012), Spatial distribution and spectral characteristics of energetic electrons in Mercury's magnetosphere, J. Geophys. Res., 117, A00M04, doi:10.1029/2012JA017983.

Hubert, D., C. Lacombe, C. C. Harvey, M. Moncuquet, C. T. Russell, and M. F. Thomsen (1998), Nature, properties, and origin of low-frequency waves from an oblique shock to the inner magnetosheath, J. Geophys. Res., 103(A11), 26783-26798, doi:10.1029/98JA01011.

Krauss-Varban, D., and N. Omidi (1991), Structure of medium Mach number quasi-parallel shocks: Upstream and downstream waves, J. Geophys. Res., 96(A10), 17715-17731, doi:10.1029/91JA01545. Le, G., P. J. Chi, X. Blanco-Cano, S. Boardsen, J. A. Slavin, and B. J. Anderson (2013), Upstream ultra-low frequency waves in Mercury's foreshock region: MESSENGER magnetic field observations, J. Geophys. Res. Space Physics, 118, 2809-2823, doi:10.1002/jgra.50342.

Liu, Y.C..-M., M. A. Lee, and H. Kucharek (2005), A quasilinear theory of ion "thermalization" and wave excitation downstream of Earth's bow shock, J. Geophys. Res., 110, A09101, doi:10.1029/2005JA011096.

Marsch, E., K.-H. Mühlhäuser, H. Rosenbauer, R. Schwenn, and F. M. Neubauer (1982a), Solar wind helium ions: Observations of the Helios solar probes between 0.3 and $1 \mathrm{AU}$, J. Geophys. Res., 87( A1), 35-51, doi:10.1029/JA087iA01p00035. 
Marsch, E., K.-H. Mühlhäuser, R. Schwenn, H. Rosenbauer, W. Pilipp, and F. M. Neubauer (1982b), Solar wind protons: Three-dimensional velocity distributions and derived plasma parameters measured between 0.3 and 1 AU, J. Geophys. Res., 87( A1), 52-72, doi:10.1029/JA087iA01p00052.

McKean, M. E., N. Omidi, and D. Krauss-Varban (1995), Wave and ion evolution downstream of quasiperpendicular bow shocks, J. Geophys. Res., 100(A3), 3427-3437, doi:10.1029/94JA02529.

Paschmann, G., N. Sckopke, S. J. Bame, and J. T. Gosling (1982), Observations of gyrating ions in the foot of the nearly perpendicular bow shock. Geophysical Research Letters, 9: 881-884. doi:10.1029/GL009i008p00881

Russell, C. T., S. S. Mayerberger, and X. Blanco-Cano (2006), Proton cyclotron waves at Mars and Venus, Adv. Space Res., 38, 745-751.

Schwartz, S. J., D. Burgess, and J. J. Moses (1996), Low-frequency waves in the Earth's magnetosheath: present status, Ann. Geophys., 14, 1134-1150.

Sckopke, N., G. Paschmann, S. J. Bame, J. T. Gosling, and C. T. Russell (1983), Evolution of ion distributions across the nearly perpendicular bow shock: Specularly and non-specularly reflected-gyrating ions, J. Geophys. Res., 88(A8), 6121-6136, doi:10.1029/JA088iA08p06121.

Shan, L., Q. Lu, M. Wu, X. Gao, C. Huang, T. Zhang, and S. Wang (2014), Transmission of large-amplitude ULF waves through a quasi-parallel shock at Venus, J. Geophys. Res. Space Physics, 119,237-245, doi:10.1002/2013JA019396.

Slavin, J. A., et al. (2008), Mercury's magnetosphere after MESSENGER's first flyby, Science, 321, 85-89, doi:10.1126/science.1159040.

Slavin, J. A., et al. (2009), MESSENGER observations of magnetic reconnection in Mercury's magnetosphere, Science, 324, 606-610.

Slavin, J.A., et al. (2010), MESSENGER observations of extreme loading and unloading of Mercury's magnetic tail, Science, 329, 665-668.

Slavin, J. A., G. A. DiBraccio, D. J. Gershman, S. Imber, G. K. Poh, J. Raines, T. H. Zurbuchen, X. Jia, D. N. Baker, S. A. Boardsen, T. Sundberg, A. Masters, C. L. Johnson, R. M. Winslow, B. J. Anderson, H. Korth, G. Ho, S. M. Krimigis, R. L. McNutt, Jr, and S. C. Solomon (2014), MESSENGER Observations of Mercury's Dayside Magnetosphere Under Extreme Solar Wind Conditions, J. Geophys. Res. Space Physics, 119, doi:10.1002/2014JA020319.

Soucek, J., C. P. Escoubet, and B. Grison (2015), Magnetosheath plasma stability and ULF wave occurrence as a function of location in the magnetosheath and upstream bow shock parameters, J. Geophys. Res. Space Physics, 120, 2838-2850, doi:10.1002/2015JA021087. 
Sundberg, T., S. A. Boardsen, J. A. Slavin, B. J. Anderson, H. Korth, T. H. Zurbuchen, J. M. Raines, and S. C. Solomon (2012a), MESSENGER orbital observations of large-amplitude Kelvin-Helmholtz waves at Mercury's magnetopause, J. Geophys. Res., 117, A04216, doi:10.1029/2011JA017268.

Sundberg, T., et al. (2012b), MESSENGER observations of dipolarization events in Mercury's magnetotail, J. Geophys. Res., 117, A00M03, doi:10.1029/2012JA017756.

Sundberg, T., S. A. Boardsen, J. A. Slavin, V. M. Uritsky, B. J. Anderson, H. Korth, D. J. Gershman, J. M.

Raines, T.H. Zurbuchen, and S. C. Solomon (2013), Cyclic reformation of a quasi-parallel bow shock at Mercury: MESSENGER observations, J. Geophys. Res. Space Physics, 118, 6457-6464, doi:10.1002/jgra.50602.

Uritsky, V. M., J. A. Slavin, G. V. Khazanov, E. F. Donovan, S. A. Boardsen, B. J. Anderson, and H. Korth (2011), Kinetic-scale magnetic turbulence and finite Larmorradius effects at Mercury, J. Geophys. Res., 116, doi:10.1029/2011JA016744.

Winslow, R. M., B. J. Anderson, C. L. Johnson, J. A. Slavin, H. Korth, M. E. Purucker, D. N. Baker, and S. C.

Solomon (2013), Mercury's magnetopause and bow shock from MESSENGER Magnetometer observations, J. Geophys. Res. Space Physics, 118, 2213-2227, doi:10.1002/jgra.50237.

Yoon, P. H., and J. Seough (2012), Quasilinear theory of anisotropy-beta relation for combined mirror and proton cyclotron instabilities, J. Geophys. Res., 117, A08102, doi:10.1029/2012JA017697.

Zwan, B. J., and R. A. Wolf (1976), Depletion of solar wind plasma near a planetary boundary, J. Geophys. Res., 81(10), 1636-1648, doi:10.1029/JA081i010p01636.

Figure 1

Four MESSENGER orbit trajectories in the noon-midnight ( $a$ and $c$ ) and dawn-dusk planes ( $b$ and $d$ ). The figures also show the average magnetopause and bow shock positions in the respective plane. From Boardsen et al. [2012].

Figure 2: An overview of the wave distribution. Each dot represents the average PSD for one wave event as a function of the ratio between the parallel and the perpendicular components of the PSD. The dashed lines represent the boundaries used to constrain the EMIC wave population. Left-hand polarized waves are marked in blue, and right-handed waves in red. 
Figure 3 - Wave characteristics for a typical magnetosheath EMIC event. The figure shows the spectral properties observed in the magnetosheath, bounded by the outermost magnetopause crossing on the left and the innermost bow shock on the right. From top to bottom, the panels show the perpendicular and parallel power spectral densities relative to the background magnetic field, the ellipticity, and the coherence. The white line marks the local proton cyclotron frequency.

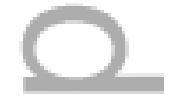

Figure 4: Magnetic field observations of EMIC waves on 19 June 2011. The left-hand panels shows the field-parallel (top panel), perpendicular (middle panels) and absolute magnetic field components (bottom panel) for an excerpt of the wave train. The right-hand panels shows hodograms of the same data period.

Figure 5: An overview of the large amplitude fluctuations seen in Mercury's magnetosheath on 22 Jun 2011. The figure shows, from top to bottom, the $x, y, z$, and absolute magnetic field components. The dashed lines mark the approximate location of the bow shock on the left, and the magnetopause on the right.

Figure 6: A detailed view of the 22 Jun 2011 event. The magnetic field data is here given in its maximum $\left(B_{1}\right)$, intermediate $\left(B_{2}\right)$ and minimum $\left(B_{3}\right)$ variance components. The hodograms associated with the three components are shown in the panels on the right.

Figure 7: Frequency spectrum of right-hand polarized waves. The distribution shows a sharp peak below the ion cyclotron frequency and a broader peak at higher frequencies.

Figure 8: Wave event on October 15, 2011. The figure follows the same format as Figure 3. The outermost magnetopause crossing and the innermost bow shock defines the left-hand and right-hand boundaries of the plot, respectively.

Figure 9: In the upper panels, the dark gray bars show the occurrence distribution of EMIC (left) and largeamplitude waves (right) as a function of the $\theta_{\mathrm{BN}}$ at the local bow shock, and the light gray background trace shows the $\theta_{B N}$ distribution of all magnetosheath passages for the time period analyzed. The bottom panels show the occurrence distributions within the magnetosheath for the EMIC (left) and largeamplitude waves (right). The spatial distribution for each magnetosheath passage have here been normalized so that the magnetopause crossing (MP) is on the left and the bow shock crossing (BS) on the right. 
Figure 10: The occurrence distribution (left) and power-spectral density (right) of the large-amplitude waves are shown as a function of the angle between the $k$-vector and the local bow shock normal, $\theta_{\mathrm{kN}}$, and the angle between the $k$-vector and the background magnetic field, $\theta_{k B}$, respectively. The figure shows that the wave normal is nearly aligned with the bow shock normal and that the closer the observations are to the exactly parallel shock, the larger the wave power.

=

(
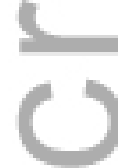

()
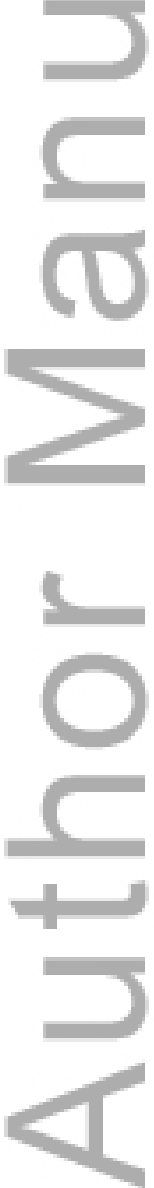

This article is protected by copyright. All rights reserved. 
$2011091(04 / 01) 0.37 \mathrm{AU}$

$2011119(04 / 29) 0.47 \mathrm{AU}$

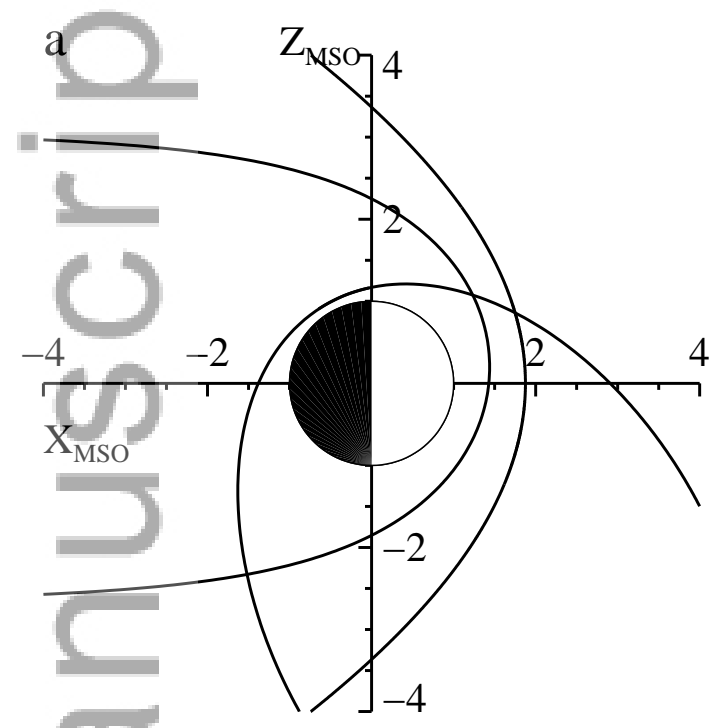

$\mathrm{b}$

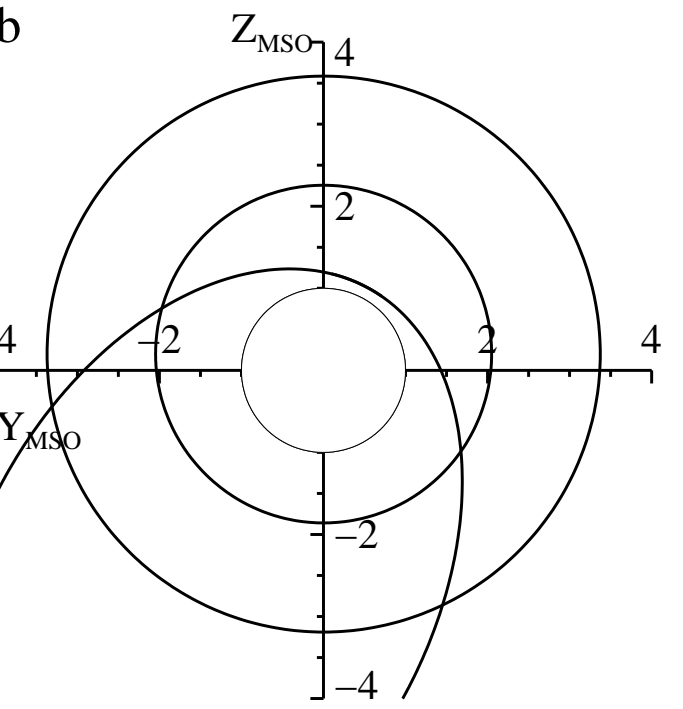

$2011146(05 / 26) 0.37 \mathrm{AU}$

$2011162(06 / 11) 0.31 \mathrm{AU}$
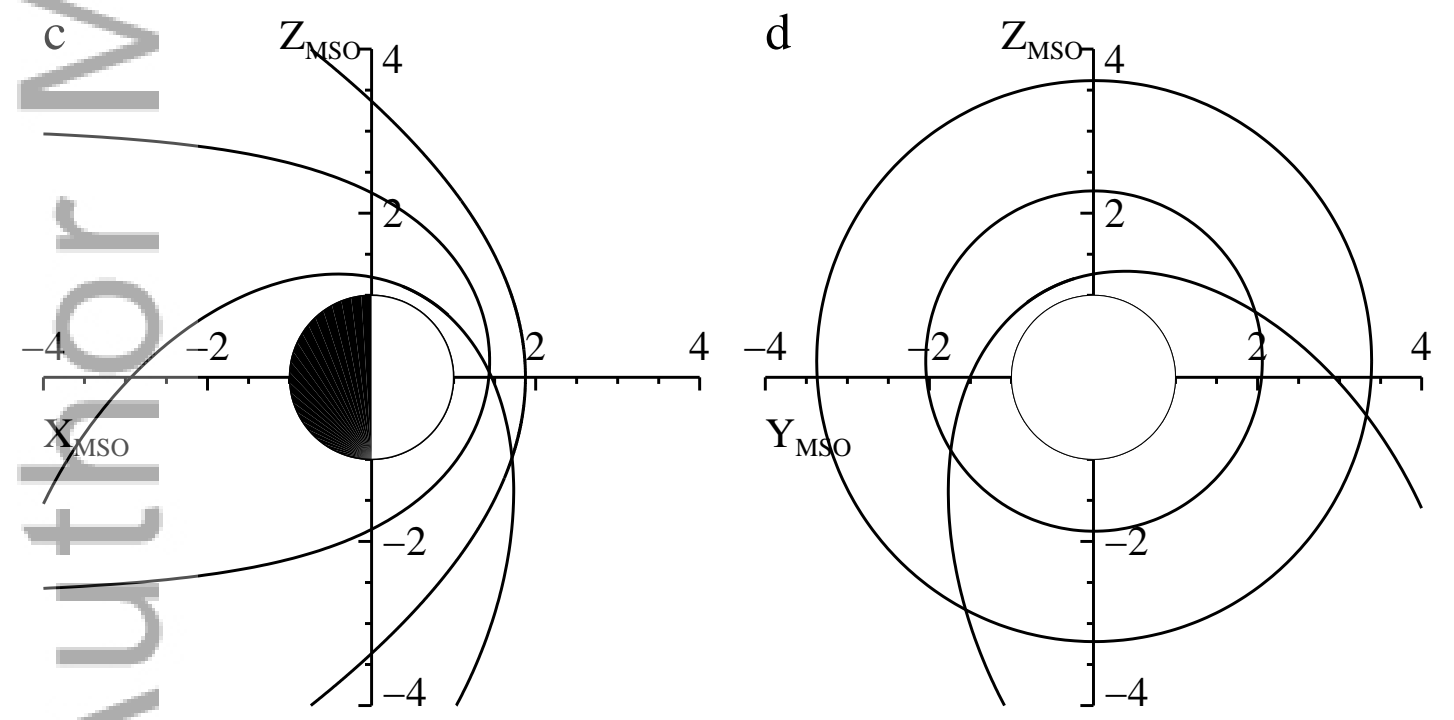


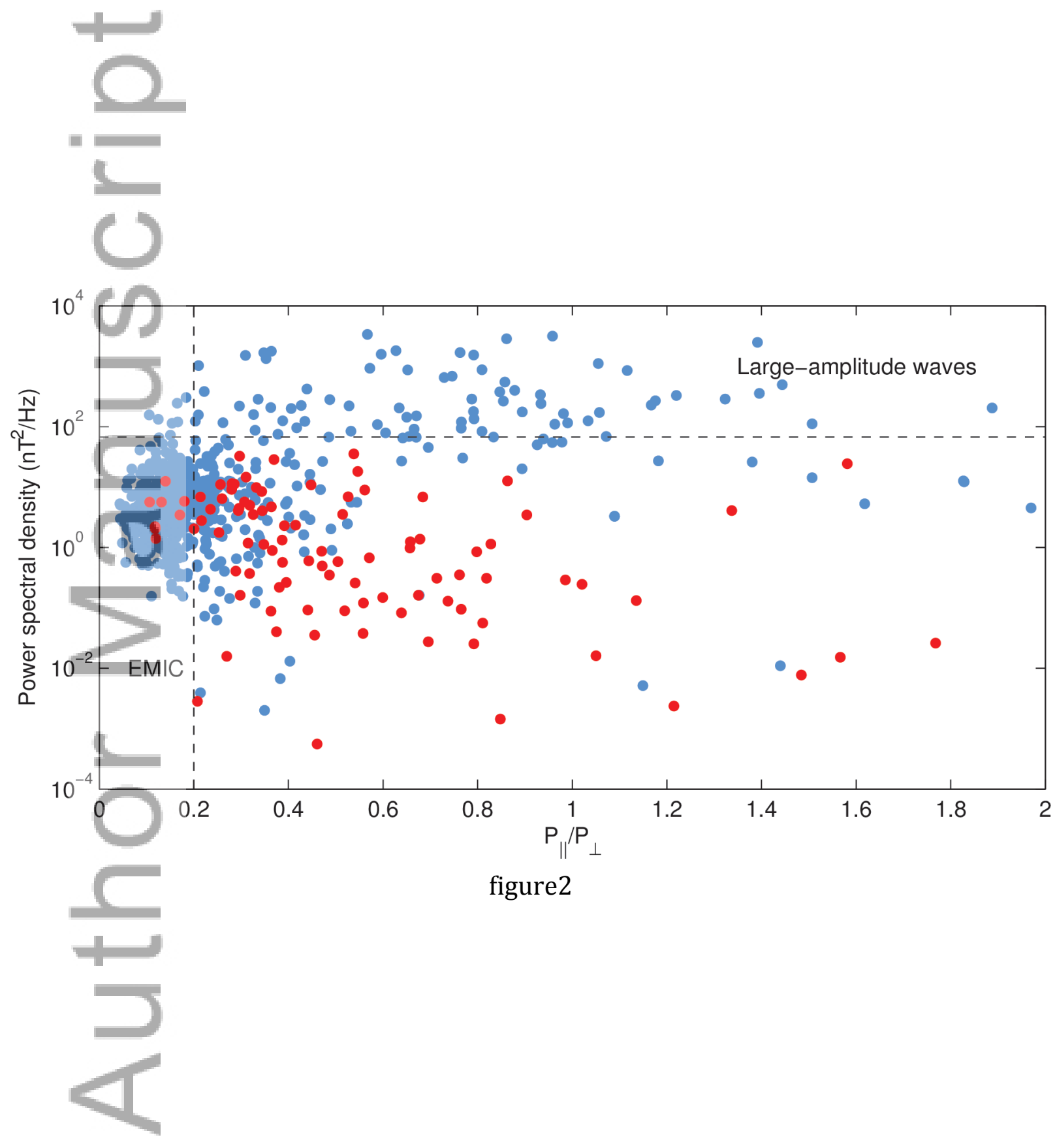

This article is protected by copyright. All rights reserved. 


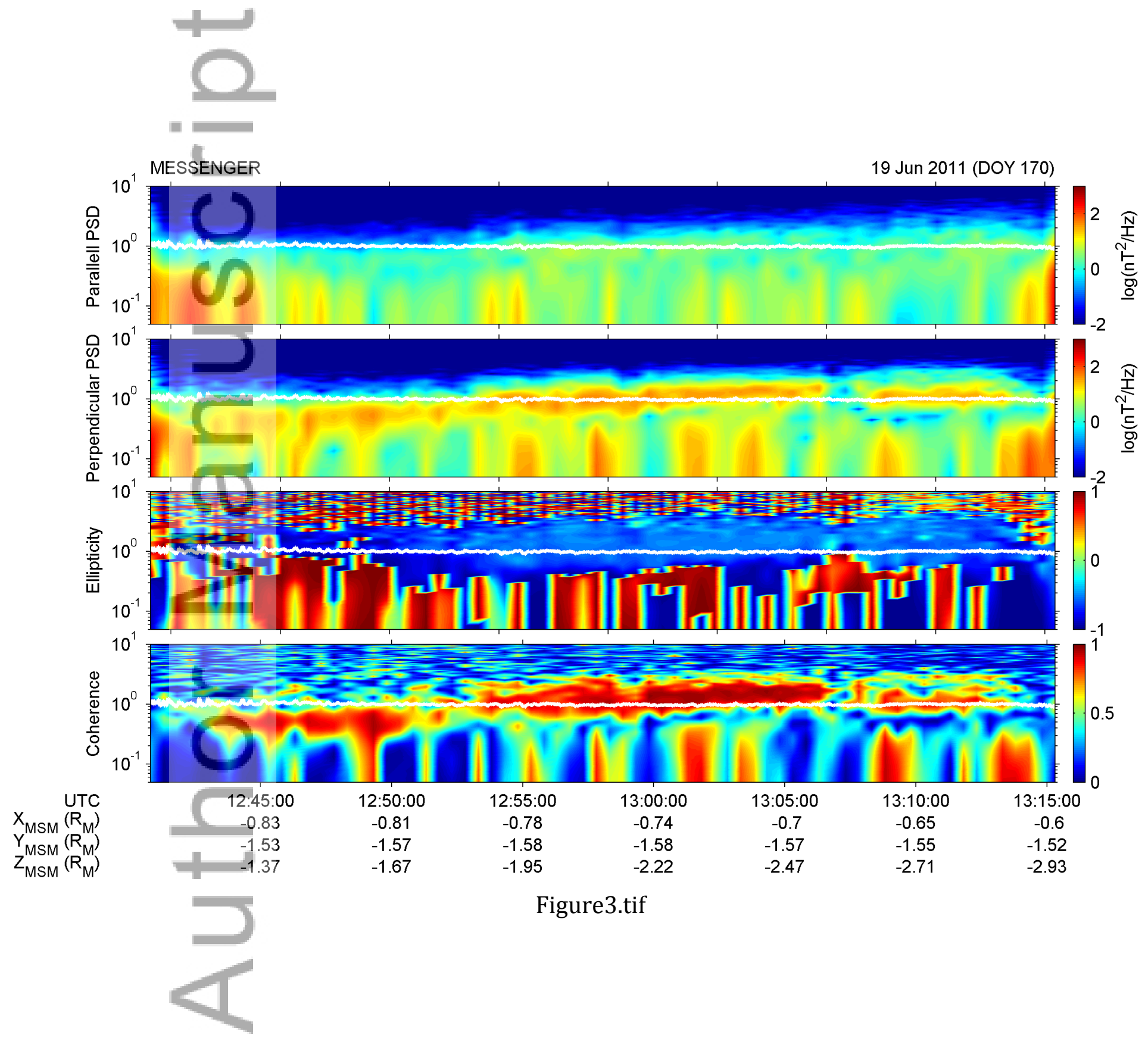

This article is protected by copyright. All rights reserved. 

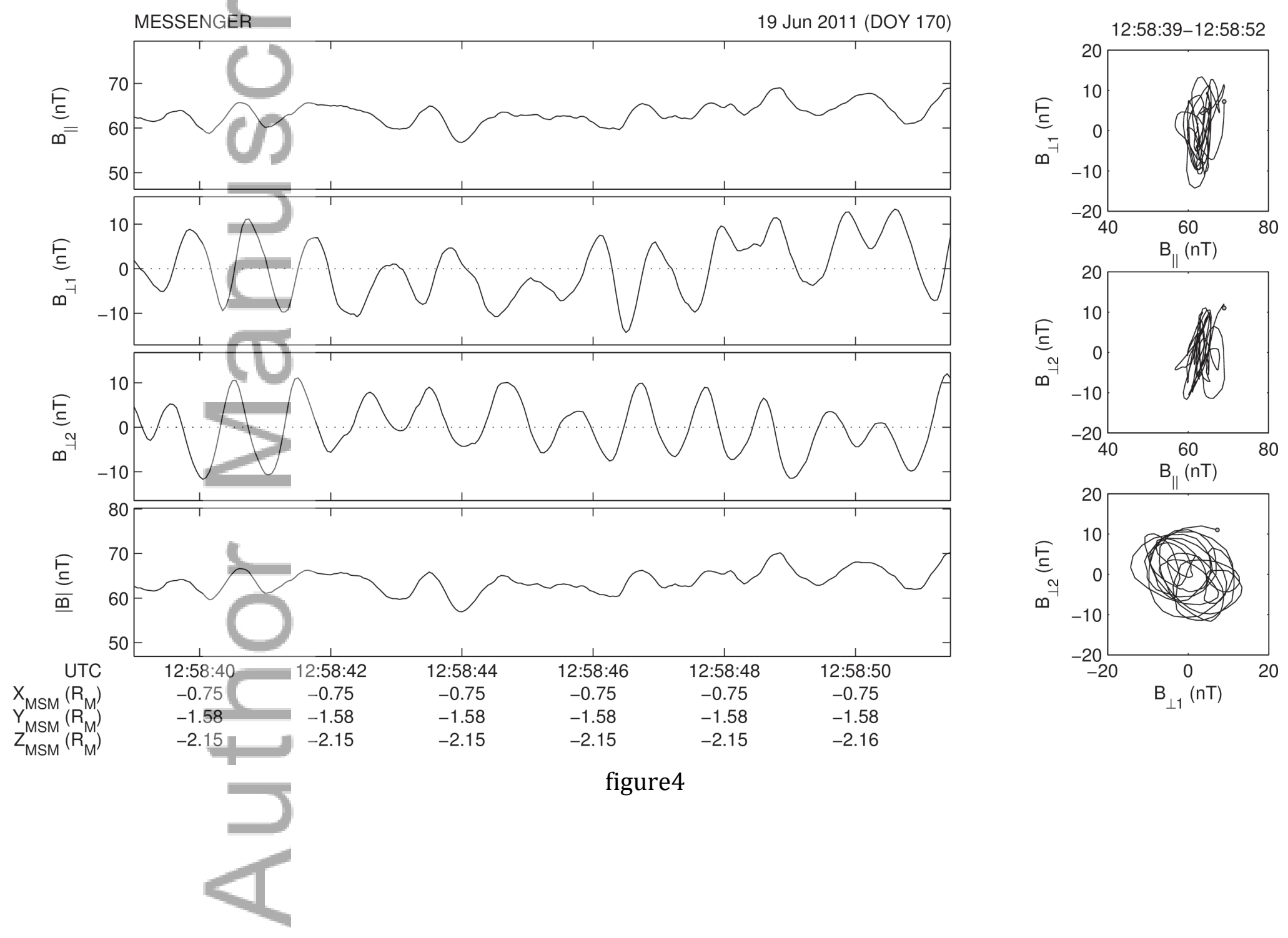

This article is protected by copyright. All rights reserved. 


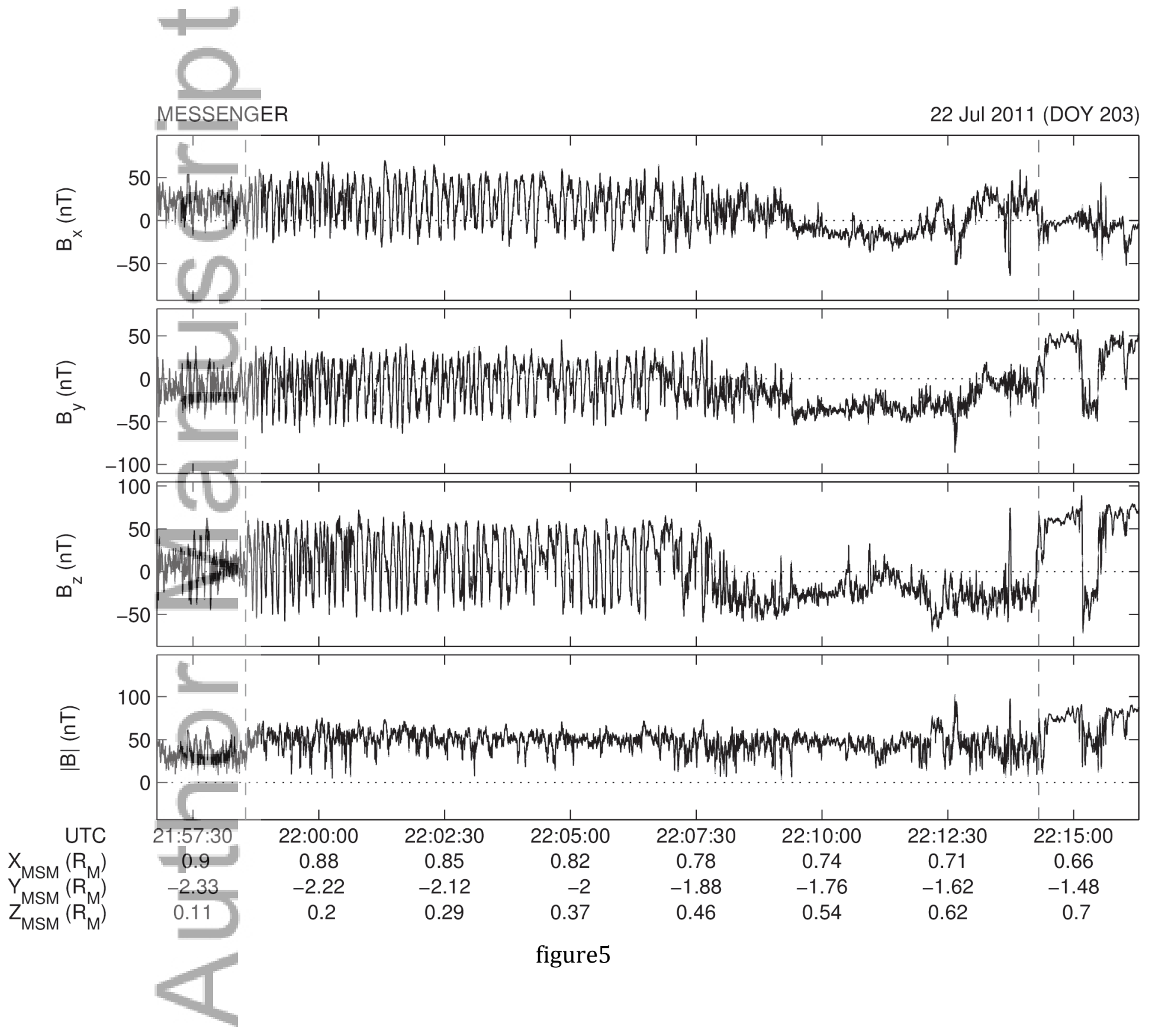

This article is protected by copyright. All rights reserved. 


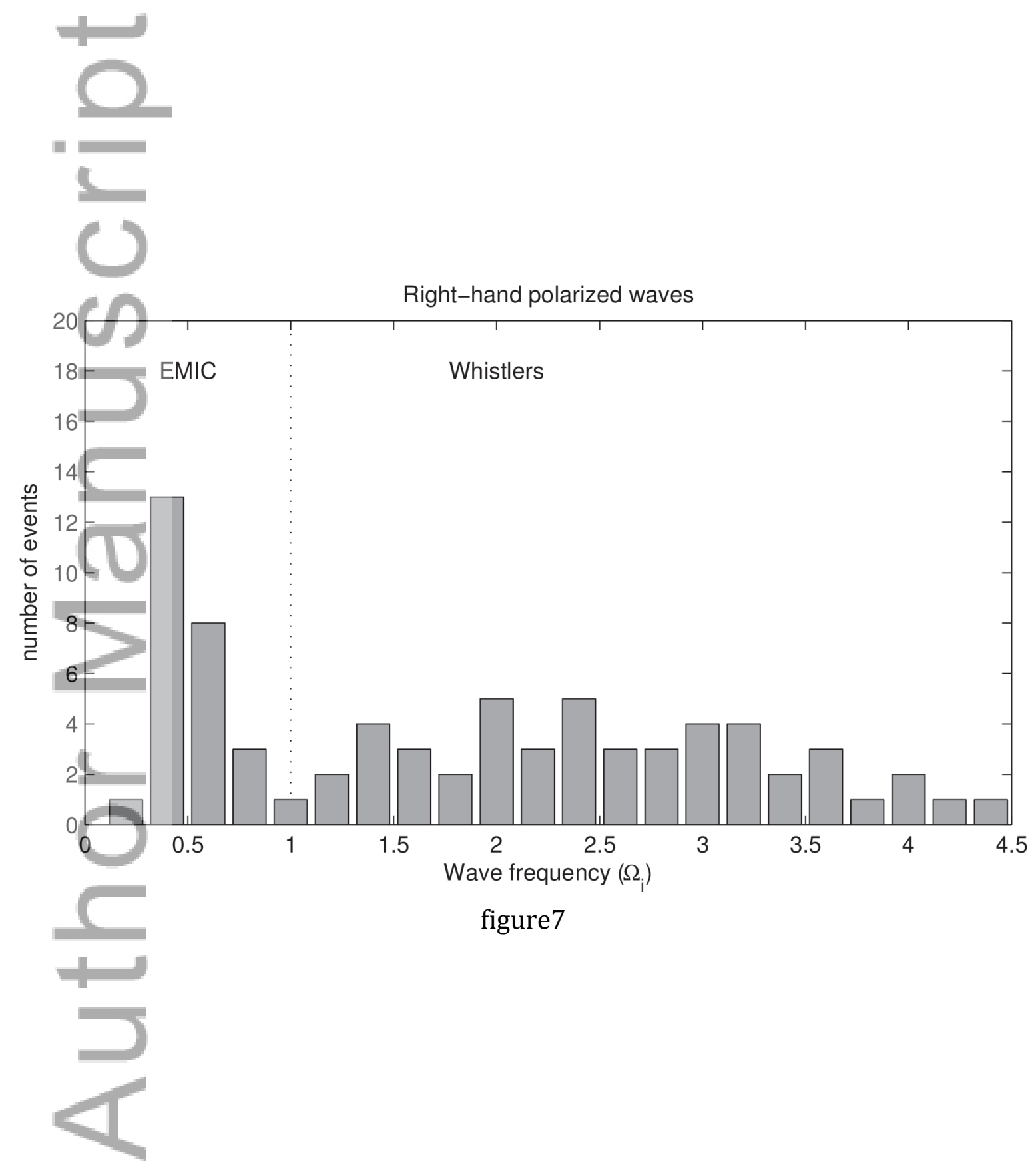

This article is protected by copyright. All rights reserved. 


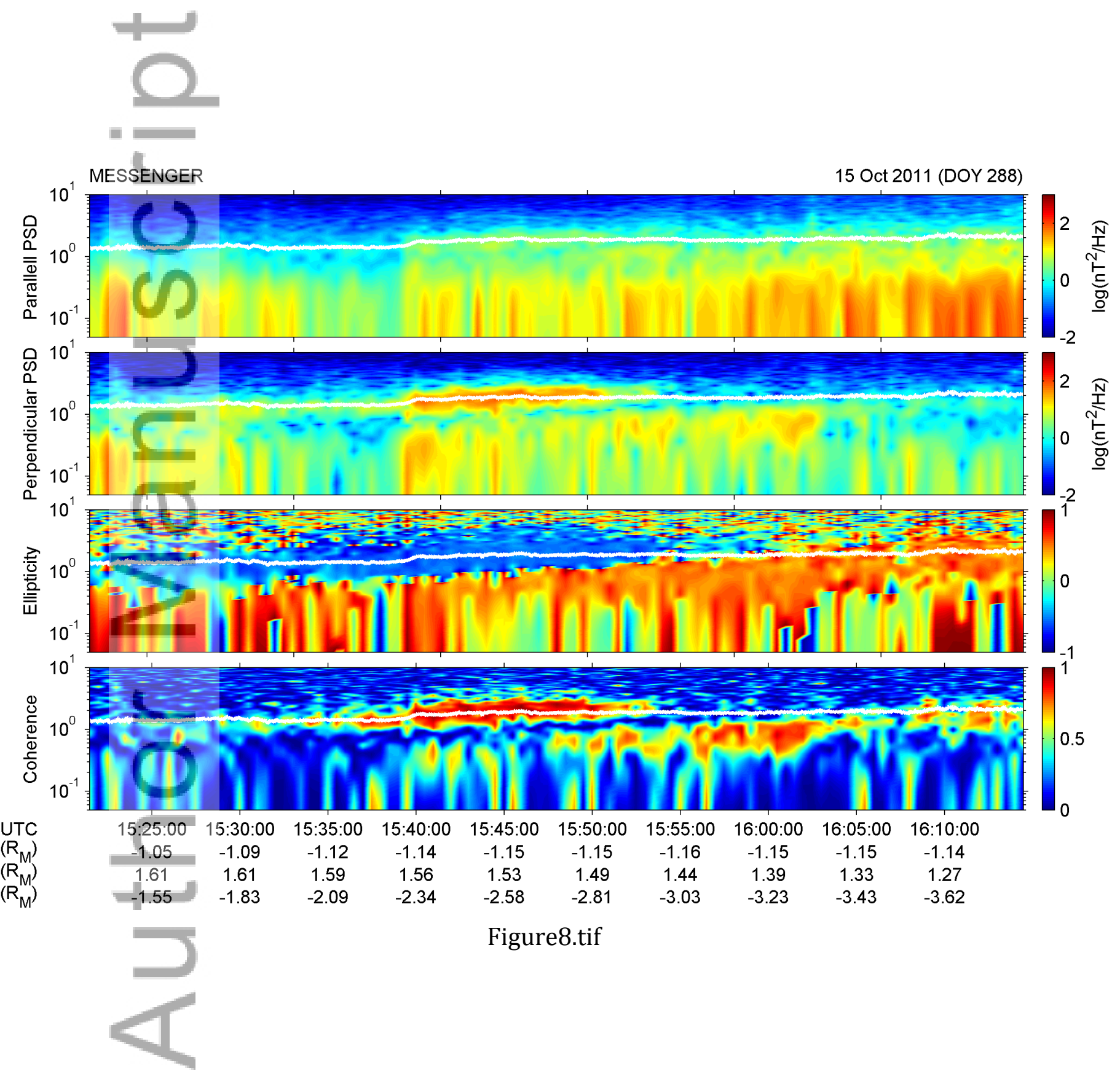

This article is protected by copyright. All rights reserved. 

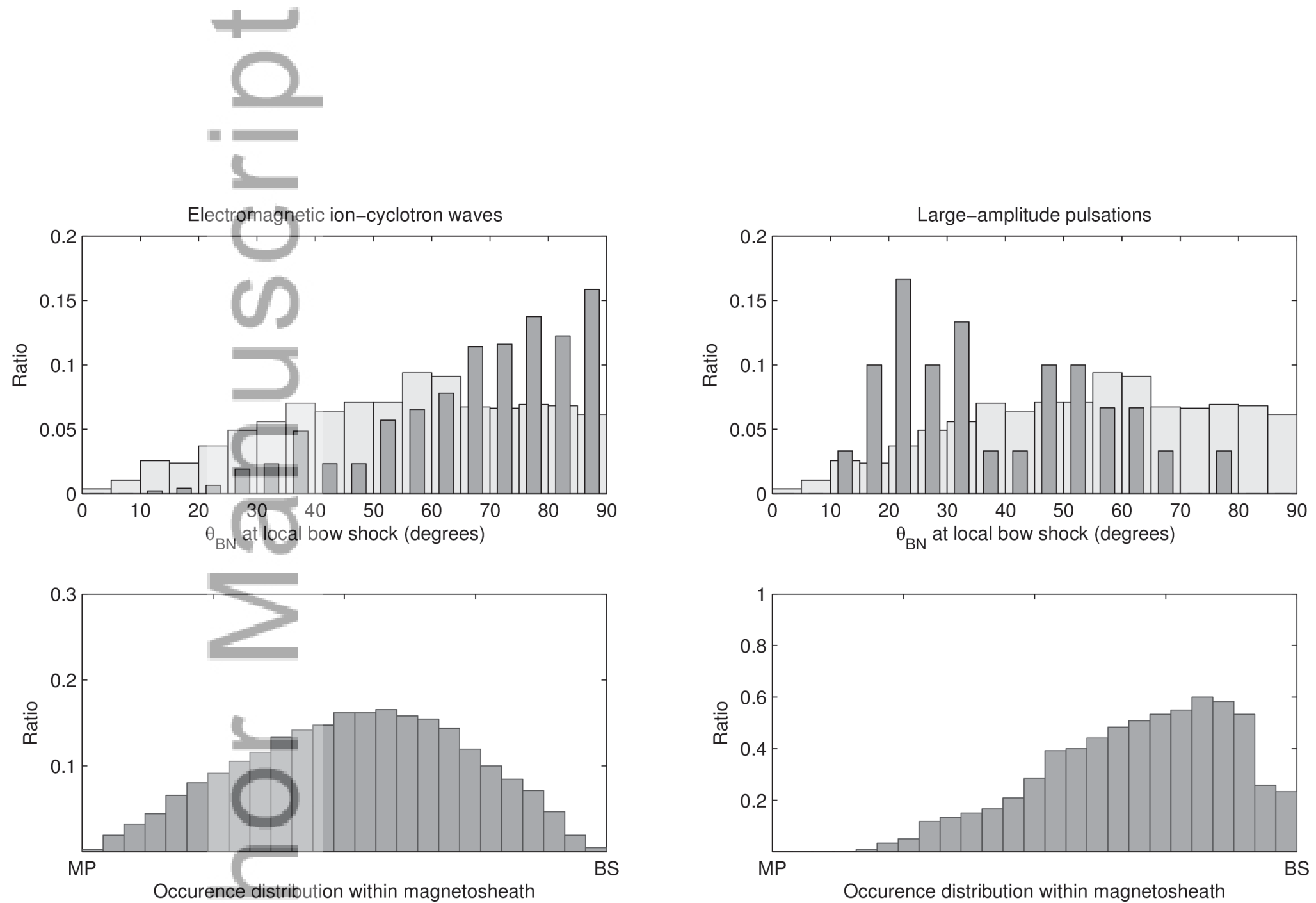

figure 9

This article is protected by copyright. All rights reserved. 

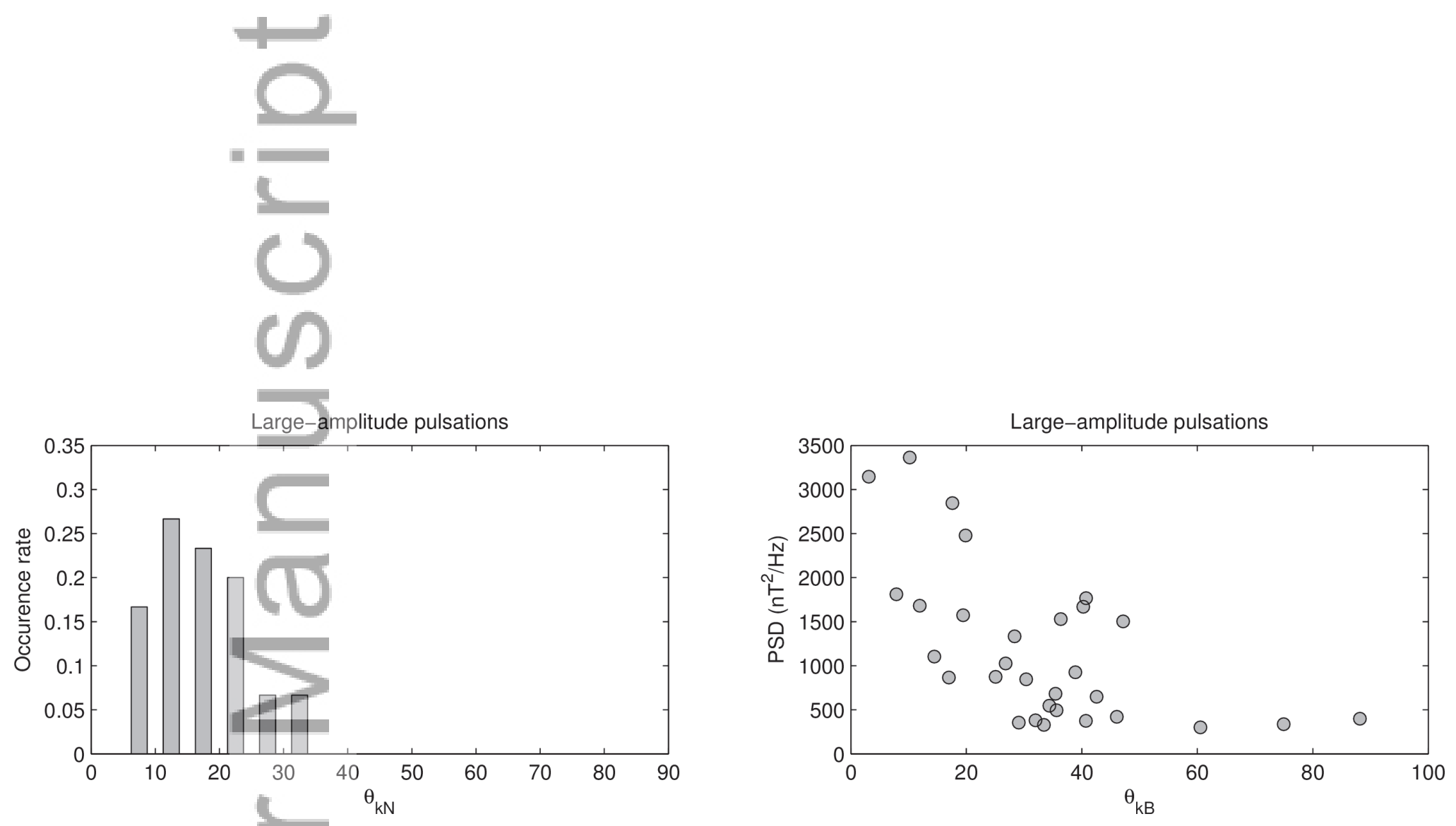

figure10

This article is protected by copyright. All rights reserved. 